\title{
PicoTesla magnetic tunneling junction sensors integrated with double staged magnetic flux concentrators
}

Guanyang He, Yiou Zhang, Lijuan Qian, Gang Xiao, Qiang Zhang, J. Carlos Santamarina, Tadeusz W. Patzek, and Xixiang Zhang

Citation: Appl. Phys. Lett. 113, 242401 (2018); doi: 10.1063/1.5052355

View online: https://doi.org/10.1063/1.5052355

View Table of Contents: http://aip.scitation.org/toc/apl/113/24

Published by the American Institute of Physics

\section{Articles you may be interested in}

Atomic-scale structure characteristics of antiferroelectric silver niobate

Applied Physics Letters 113, 242901 (2018); 10.1063/1.5061749

Compact acoustic double negative metamaterial based on coexisting local resonances

Applied Physics Letters 113, 244101 (2018); 10.1063/1.5052026

Measuring the orientation of the flexural vibrations of a cantilevered microwire with a micro-lens fiber-optic interferometer

Applied Physics Letters 113, 243101 (2018); 10.1063/1.5021801

Multilevel resistive and magnetization switching in $\mathrm{Cu} / \mathrm{CoFe}{ }_{2} \mathrm{O}_{4} / \mathrm{Pt}$ device: Coexistence of ionic and metallic conducting filaments

Applied Physics Letters 113, 243501 (2018); 10.1063/1.5043078

Reduced interfacial magnetic moment of $\mathrm{Y}_{3} \mathrm{Fe}_{5} \mathrm{O}_{12}$ by capping $\mathrm{Pt}$

Applied Physics Letters 113, 182402 (2018); 10.1063/1.5046763

Passive ultrasound aided acoustic resolution photoacoustic microscopy imaging for layered heterogeneous media

Applied Physics Letters 113, 241901 (2018); 10.1063/1.5064417

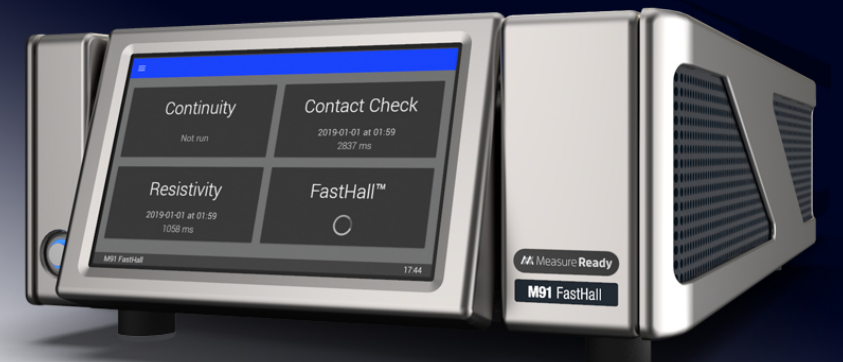

\section{Measure Ready M91 FastHall ${ }^{\mathrm{TM}}$ Controller \\ A revolutionary new instrument for complete Hall analysis}




\title{
PicoTesla magnetic tunneling junction sensors integrated with double staged magnetic flux concentrators
}

\author{
Guanyang He, ${ }^{1}$ Yiou Zhang, ${ }^{1}$ Lijuan Qian, ${ }^{1}$ Gang Xiao, ${ }^{1, a)}$ Qiang Zhang, ${ }^{2}$ \\ J. Carlos Santamarina, ${ }^{2}$ Tadeusz W. Patzek, ${ }^{2}$ and Xixiang Zhang ${ }^{2}$ \\ ${ }^{1}$ Department of Physics, Brown University, Providence, Rhode Island 02912, USA \\ ${ }^{2}$ Division of Physical Science and Engineering, King Abdullah University of Science and Technology, \\ Thuwal 239955, Kingdom of Saudi Arabia
}

(Received 16 August 2018; accepted 21 November 2018; published online 10 December 2018)

\begin{abstract}
Ultra-sensitive solid-state magnetic sensors are in strong demand in many applications where currently available sensors are inadequate. We have used high performance magnetic tunneling junction (MTJ) sensors and pushed the magnetic sensing limit to a high level. We have incorporated double-staged magnetic flux concentrators, one on the MTJ chip level and the other on a more macroscopic level, to amplify the external field of interest. With this approach and undergoing a process of optimization on the flux concentrators, we have increased the sensitivity of the MTJ sensor by a large factor of 517 to $775.4 \% / \mathrm{Oe}$ in terms of magnetoresistance response. The coercivity of the sensor is only $0.12 \mathrm{Oe}$. We have achieved a detectable field limit of $30 \mathrm{pT} / \sqrt{\mathrm{Hz}}$ at $10 \mathrm{kHz}$. We have presented the noise spectrum and the sensitivity spectrum up to a maximum frequency of $100 \mathrm{kHz}$. Published by AIP Publishing. https://doi.org/10.1063/1.5052355
\end{abstract}

Based on the spin-dependent quantum tunneling effect, magnetic tunneling junctions (MTJs) have been developed into a high performance solid-state magnetic sensor for their superior properties, including high sensitivity, low power consumption, miniaturized size, thermal stability, broad frequency response, and operational tunability. ${ }^{1-6}$ The high magnetoresistance ratio (MR) is a particularly valuable property which allows the MgO-based MTJs to generate large signals in response to a weak external magnetic field. Scientists have been making efforts in minimizing the intrinsic magnetic noise of the MTJs, so that the signal-to-noise ratio (SNR) can be enhanced as much as possible. ${ }^{7-14}$ The journey to increase magnetic sensitivity is both arduous and rewarding. Every milestone reached brings new and challenging applications into reality. For example, MTJs are the sensor of choice for read-write heads in data storage devices ${ }^{15}$ and for magnetic imaging, ${ }^{16}$ microscopy, ${ }^{17}$ and metrology. ${ }^{18}$ Precise magnetoresistive sensors are used in underground navigation and resource (oil and gas) exploration. ${ }^{19,20}$ MTJs may also be adapted for bio-medical applications, such as bio-molecular recognition. ${ }^{21,22}$

In this work, we aim to develop robust and easy-to-use MTJ sensors with picoTesla (pT) field sensitivity by researching on extrinsic magnetic flux concentrators (MFCs), which amplify external magnetic fields. Our MFCs consist of two stages, onchip MFCs (onMFCs) and external MFCs (exMFCs). We design and process two types of "soft" magnetic materials with high permeability and low coercivity $\left(H_{c}\right)$, a thin film for the onMFC and a bulk alloy for exMFC. We achieve a flux amplification of a few hundred-fold, and picoTesla (pT)-scale field sensitivity over a broad frequency range up to $100 \mathrm{kHz}$.

Highly sensitive magnetic sensors rely on the fabrication of high quality MTJ wafer stacks, which we prepared by using a home-made high vacuum magnetron sputtering

${ }^{a)}$ Email: Gang_Xiao@brown.edu system with a base pressure of $2 \times 10^{-8}$ Torr. We deposited the MTJ stacks on 2-in. thermally oxidized silicon wafers according to the layer sequence of Si-substrate $/ \mathrm{SiO}_{2} / \mathrm{Ta}(50) /$ $\mathrm{Ru}(300) / \mathrm{Ta}(50) / \mathrm{Co}_{50} \mathrm{Fe}_{30}(30) / \mathrm{IrMn}(180) / \mathrm{Co}_{50} \mathrm{Fe}_{30}(30) / \mathrm{Ru}(8.5) /$ $\mathrm{Co}_{40} \mathrm{Fe}_{40} \mathrm{~B}_{20}(30) / \mathrm{MgO}(25) / \mathrm{Co}_{40} \mathrm{Fe}_{40} \mathrm{~B}_{20}(25) / \mathrm{Ta}(3) /$ Conetic(400)/ $\mathrm{Ta}(50) / \mathrm{Ru}(100)$ (numbers referring to thickness in Angstroms). We deposited the $\mathrm{MgO}$ tunneling layer under 1.2 mTorr $\mathrm{Ar}$ sputtering pressure and $120 \mathrm{~W}$ RF power, and all other layers under $2 \mathrm{mTorr}$ and DC sputtering with various powers. The bottom $\mathrm{CoFeB}$ below the $\mathrm{MgO}$ barrier is a magnetically fixed layer and the top $\mathrm{CoFeB} / \mathrm{Ta} /$ Conetic composite layer is the magnetic free layer. The Conetic ${ }^{\circledR}$ layer is a soft ferromagnetic alloy with a composition of $\mathrm{Ni}_{77} \mathrm{Fe}_{14} \mathrm{Cu}_{5} \mathrm{Mo}_{4}$, which is similar to but softer than the permalloy. ${ }^{23,24} \mathrm{We}$ used standard photolithography and physical ion milling to pattern the stacks into hundreds of magnetic sensor units on each wafer. Figure 1(a) is a schematic of an individual MTJ which has an oval shape. The fabrication process was concluded by subjecting the wafer to a magnetic annealing process at $320^{\circ} \mathrm{C}$ for $2 \mathrm{~h}$ in a high vacuum of $6 \times 10^{-7}$ Torr and under an in-plane field of $0.45 \mathrm{~T}$. How an MTJ sensor is designed geometrically and conditioned magnetically (pinned fixed electrode versus free electrode) are explained in Ref. 1. One sensor unit (a die) has a silicon footprint of $1 \times 1 \mathrm{~mm}^{2}$, consisting of four MTJs connected in series as shown in the optical micrograph of Fig. 1(b). Each MTJ has an oval shape with a dimension of $15 \times 120 \mu \mathrm{m}^{2}$. The sensor is a two-terminal resistor-like device with a typical resistance of $2.5 \mathrm{k} \Omega$ and a total MR of about $95 \%$ between the parallel and antiparallel magnetization configuration of the bottom and top magnetic electrodes. The field sensing direction is along the $\mathrm{x}-$ axis which is the short axis of the MTJ ellipse, as shown in Fig. 1(b). Two gold pads along the central y-axis serve as the two terminals of the MTJ sensor.

Now we provide the technical details of our two-stage MFC development (onMFC and exMFC). The first stage onMFC consists of a pair of trapezoids made of $\mathrm{Co}_{88} \mathrm{Zr}_{4} \mathrm{Nb}_{8}$ 
a)

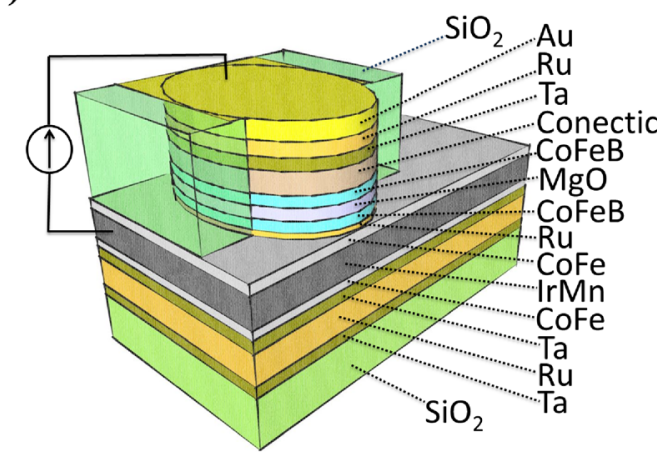

b)

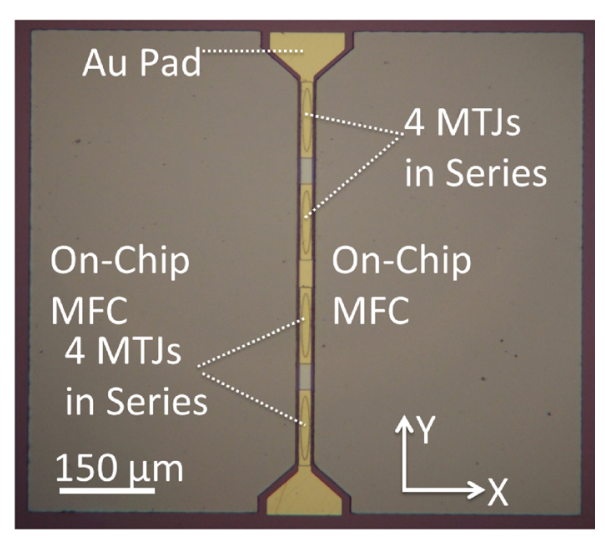

c)

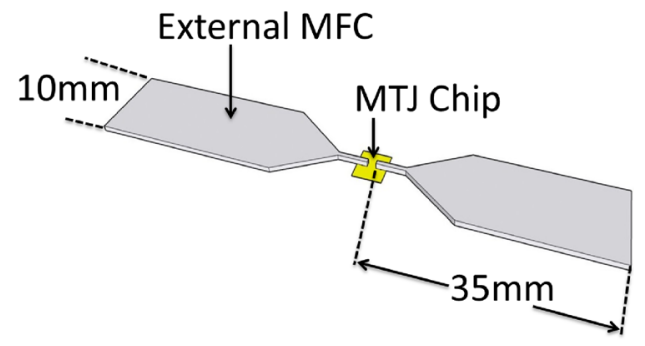

FIG. 1. (a) Schematic of the entire MTJ layer structure and patterned oval MTJ etched to the bottom of the pinned $\mathrm{CoFeB}$ layer. Ellipse long axis = $120 \mu \mathrm{m}$ and short axis $=15 \mu \mathrm{m}$ (aspect ratio 8). Pinning is along the short axis, which is also the sensing direction. The magnetization of the top "free" composite layer, $\mathrm{CoFeB} / \mathrm{Conetic}$, is along the long axis due to shape anisotropy; (b) micrograph of an MTJ sensor unit (die area $1 \times 1 \mathrm{~mm}^{2}$ ) consisting of four MTJs connected in series along the central y-axis and a pair of trapezoid-shaped on-chip flux concentrators made of the CoZrNb film. The two terminals along the $y$-axis are gold contact pads for the sensor (sensing along the $\mathrm{x}$-axis); and (c) schematic of a pair of external magnetic flux concentrators (Conetic alloy) with two tips in contact with the on-chip MFC.

( $\mathrm{CoZrNb}$ ) film which is deposited/patterned in close proximity to the MTJ sensor unit, as can be seen in Fig. 1(b). The central gap between the two onMFCs is $30 \mu \mathrm{m}$, and the external field in the sensing direction is amplified within the gap. $\mathrm{CoZrNb}$ is a soft magnetic material with a high permeability $\mu$ varying from 1000 to 7000 as reported in the literature..$^{25-29}$ It is an ideal MFC material for solid-state magnetic sensors. ${ }^{30,31}$ Following previous studies, ${ }^{32,33}$ we deposited a CoZrNb amorphous thin film using a 99.95\%-purity sputtering target. To optimize its growth condition, we first deposited a $\mathrm{CoZrNb}$ film on oxidized silicon wafers and measured its magnetic properties using vibrating sample magnetometry (VSM). After many trials and errors, we obtained the best sputtering conditions for optimized "soft" magnetic properties (high permeability and low coercivity): base vacuum pressure $1.8 \times 10^{-7}$ Torr; Ar gas sputtering pressure $5.0 \times 10^{-4}$ Torr; DC sputtering power $100 \mathrm{~W}$; and $\mathrm{CoZrNb}$ film thickness $1200 \mathrm{~nm}$. The purity of the material, high vacuum level, moderate sputtering power, as well as the low sputtering pressure seem to be necessary to obtain "softness" of CoZrNb. Figure 2(a) shows a representative magnetic hysteresis loop for a $1200 \mathrm{~nm}$-thick CoZrNb film. The saturation magnetization is about $950 \mathrm{emu} / \mathrm{cm}^{3}$. The saturation field is about $4 \mathrm{Oe}$ and $H_{c}=0.35 \mathrm{Oe}$. The magnetic permeability is 3282 .

Patterning the continuous $\mathrm{CoZrNb}$ film into onMFC has the tendency to increase the $H_{c}$. The edges and the associated roughness, as well as the trapezoid shape, are some of the factors affecting the $H_{c}$. Figure 2(b) shows the linear transfer
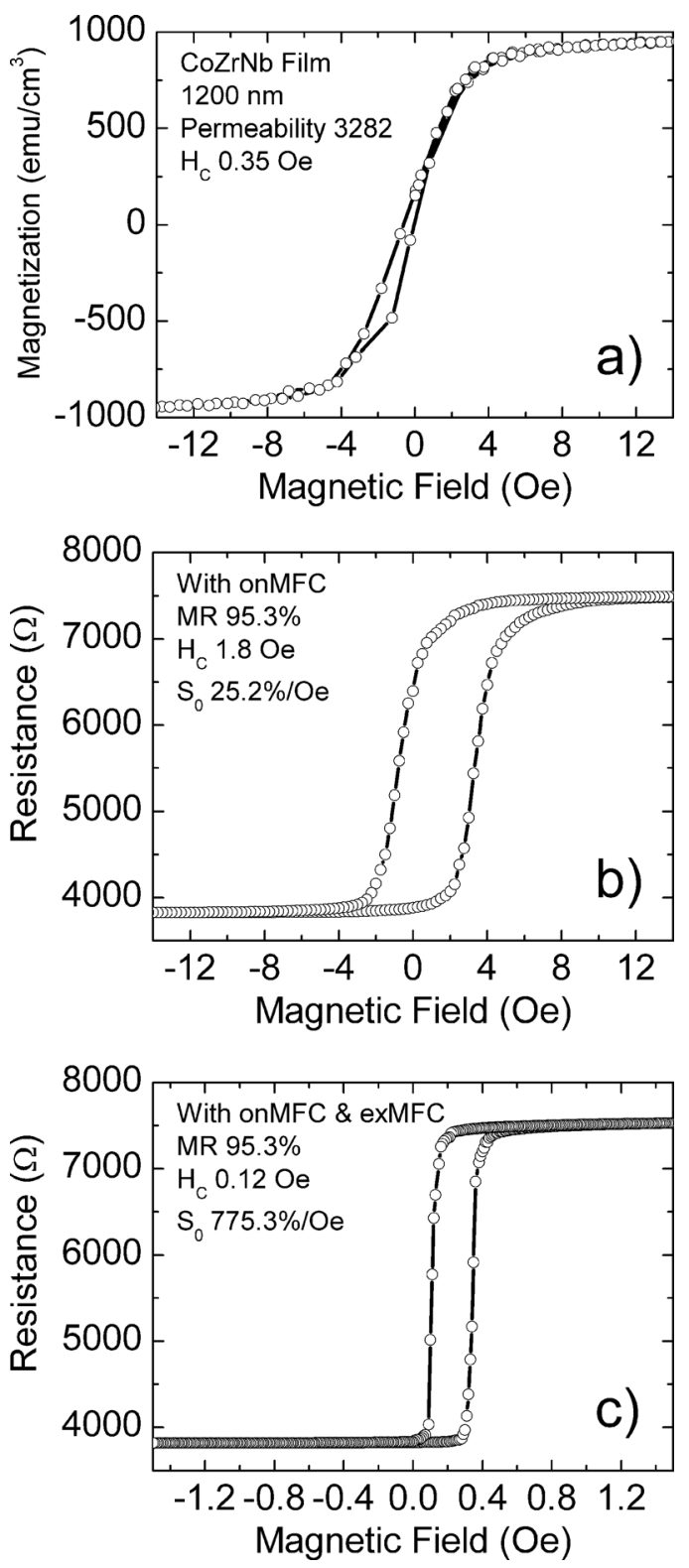

FIG. 2. (a) Magnetic hysteresis loop of the sputtered $\mathrm{CoZrNb}$ film with a thickness of $1200 \mathrm{~nm}$ at $300 \mathrm{~K}$. This film has a magnetic permeability of 3282 and a coercivity of $0.35 \mathrm{Oe}$; (b) resistance transfer curve of an MTJ sensor with integrated on-chip magnetic flux concentrators (onMFCs). The total magnetoresistance is $95.3 \%$. The coercivity is 1.8 Oe. The field sensitivity is $25.2 \% / \mathrm{Oe}$; and (c) resistance transfer curve of an MTJ sensor with dual MFCs (onMFC and exMFC). The total magnetoresistance is $95.3 \%$. The coercivity is $0.12 \mathrm{Oe}$. The field sensitivity is $775.3 \%$. 
curve (resistance versus sensing field) of a representative MTJ sensor with onMFC within \pm 14 Oe. The coercive field is $H_{C}=1.8 \mathrm{Oe}$. The magnetic field sensitivity $S_{0}$ is defined as

$$
S_{0}=\frac{1}{R_{0}} \frac{\Delta R}{\Delta H},
$$

where $R_{0}$ is the center resistance on the transition curves. We can provide a biasing magnetic field to keep the sensor at the $R_{0}$ state, where it has the highest resistance slope of $\Delta R / \Delta H$. For the sensor used for Fig. 2(b), $S_{0}{ }^{\prime}=25.2 \% / \mathrm{Oe}$. As a comparison, the MTJ sensor without onMFC carries a typical sensitivity $S_{0}$ of $1.5 \% / \mathrm{Oe}$. Therefore, the amplification factor for the onMFC is $\beta_{1}=S_{0}{ }^{\prime} / S_{0}=16.8$.

Our second stage exMFC is a pair of "macroscopic" flux concentrators, as shown schematically in Fig. 1(c). We use a bulk metallic sheet $\left(0.5 \mathrm{~mm}\right.$ thick) of the Conetic ${ }^{\circledR}$ alloy $\left(\mathrm{Ni}_{77} \mathrm{Fe}_{14} \mathrm{Cu}_{5} \mathrm{Mo}_{4}\right)$ which is annealed by the manufacturer and carries a permeability of 18.8. There are various designs of exMFC, and we adopt a similar shape given in Ref. 24 for its effectiveness. Each concentrator is shaped as a rectangle with one end reduced into a tip. The width of the rectangle and the tip is $10 \mathrm{~mm}$ and $1 \mathrm{~mm}$, respectively. The overall length of the concentrator is $35 \mathrm{~mm}$. We integrate the two concentrators with their tips facing the microscopic MTJ sensor, and the gap between the two tips is about $0.8 \mathrm{~mm}$. The tips are carefully placed onto the onMFC to avoid any air gap between the onMFC and the exMFC, therefore, reducing the magnetic flux "resistance". Figure 2(c) shows the linear transfer curve of a representative MTJ sensor with double-staged onMFC and exMFC. The field sensitivity is enhanced to $S_{0}{ }^{\prime \prime}=775.3 \% / \mathrm{Oe}$, implying the second stage exMFC sports an amplification factor of $\beta_{2}=S_{0}{ }^{\prime \prime} / S_{0}{ }^{\prime}=30.8$. The total amplification factor is $\beta=\beta_{1} \beta_{2}=516.9$. The sensor in Fig. 2(c) carries a small coercivity of only 0.12 Oe. Our double-staged MFCs generate an extremely large sensitivity, but the field dynamic range is reduced significantly. The reduction in the dynamic range is inevitable since the amplification of the external field would enable the field to saturate the sensor easily. As shown in Fig. 2(c), each sensing edge is saturated within a narrow field range of 0.1 Oe. Therefore, to operate effectively, we need to field-bias the sensor at the $R_{0}$ state under an ambient environment, e.g., the Earth's magnetic field. The large size of the exMFC makes the sensor highly sensitive, but at the cost of reduced spatial resolution, rendering the sensor more suitable for applications such as underground energy explorations.

The benefit of applying MFCs on MTJ sensors has been previously studied in Ref. 24, in which only an estimation of detectability was calculated using MTJ sensitivity for nanoTesla magnetic fields. However the sensitivity of MTJs tends to decrease dramatically for smaller magnetic fields as shown in their work. Therefore, in our work, by doing measurements directly under picoTesla magnetic fields, we characterize completely the detectable field limit, the frequency response, and the intrinsic noise of our MTJ sensor. The experimental setup is shown in Fig. 3(a). Two AAAbatteries in series with a resistor $R_{1}$ constitute a DC bias current source, applying approximately $1.5 \mathrm{~V}$ bias voltage on the MTJ sensor. A function generator (Agilent 33220A) is a)

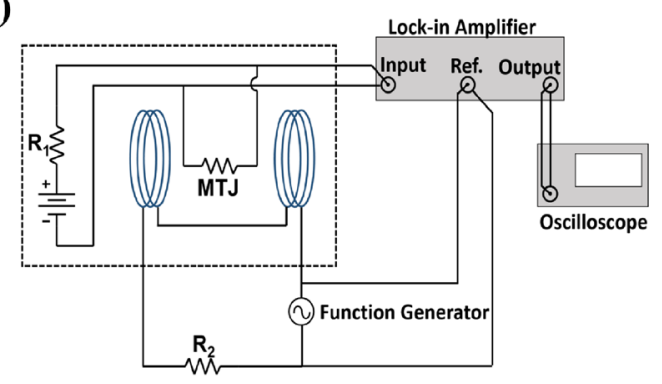

b)

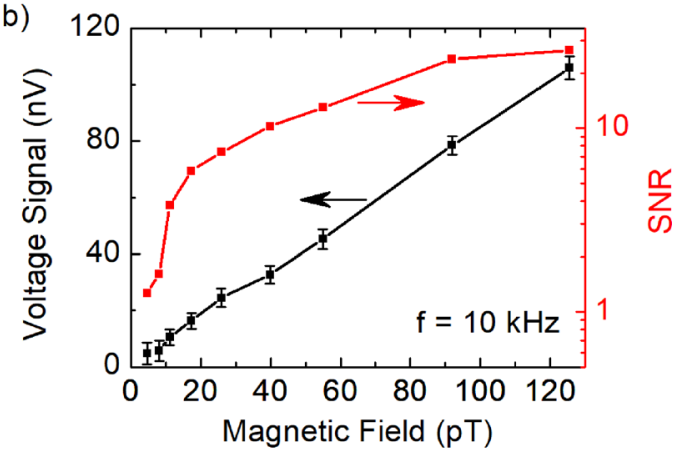

c)

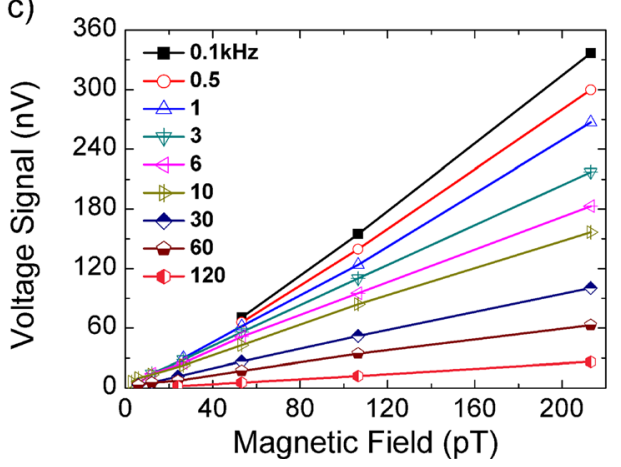

FIG. 3. (a) Experimental setup in measuring the voltage signal and noise level of MTJ sensors; output of the lock-in amplifier is recorded on an oscilloscope. The MTJ sensor, the bias current source, and the coil are enclosed in an aluminum shield box (dashed line) to reduce electromagnetic interference from the environment. (b) The voltage signal and SNR measured by the lockin amplifier as a function of the amplitude of the testing AC magnetic field at $10 \mathrm{kHz}$. (c) The voltage signal measured at different frequencies.

connected in series with a pair of calibrated coils near the sensor and a current-limiting resistor $R_{2}$, to generate an $\mathrm{AC}$ testing magnetic field. One additional coil (not shown in the figure) is used to apply a DC biasing field so that the sensor is maintained at the $R_{0}$ state. The voltage signal across the sensor is fed into a lock-in amplifier, which has a negligible FET-input voltage noise of $5 \mathrm{nV} / \sqrt{\mathrm{Hz}}$. During measurement, we set the low-pass filter of the lock-in amplifier output with a time constant of $5 \mathrm{~s}$ and a slope of $24 \mathrm{~dB} /$ octave, corresponding to an equivalent noise bandwidth of $0.024 \mathrm{~Hz}$.

When an AC testing magnetic field at a certain amplitude is applied, the oscilloscope in Fig. 3(a) would record stabilized output of the lock-in amplifier for $100 \mathrm{~s}$. The mean value of the recorded output corresponds to the sensor's voltage signal in response to the testing field, and its fluctuation, i.e., standard deviation, represents the noise level of the sensor. Figure 3(b) shows the voltage signal and the signal-tonoise ratio (SNR) as a function of the amplitude of the AC testing field at $10 \mathrm{kHz}$. As the amplitude is reduced from 213 
to $5 \mathrm{pT}$, the SNR drops to approximately 1, which reveals the detection limit of $5 \mathrm{pT}$ at $10 \mathrm{kHz}$.

Both the sensor's signal and noise can depend on the frequency of the testing magnetic field, so it is essential to determine the detectable field limit at different frequencies. Figure 3(c) shows the voltage signals of the MTJ sensor from 0.1 to $120 \mathrm{kHz}$. At each frequency, we gradually reduce the amplitude of the testing magnetic field until the SNR reaches 1 . At all frequencies, the voltage signal of such sensors scales linearly with the magnetic field, yet this response (slope of the line) decreases with increasing frequency. The soft magnetic materials of MFCs become less responsive for higher frequency, as their permeability goes down. This is, in part, due to induced eddy currents in MFCs. Moreover, the detectable field limit becomes larger at lower frequencies despite the increment in the sensor response, indicating the noise level has a strong frequency dependence.

We have also calculated the sensor's noise power spectral density at different frequencies. It is given by the ratio between the standard deviation of the voltage signal and the square root of equivalent noise bandwidth, and has no dependence on the settings of the lock-in amplifier. Figure 4(a) shows the noise of the MTJ sensor from 0.1 to $120 \mathrm{kHz}$. The noise power spectral density of each MTJ can be characterized by

$$
S_{V}\left(V^{2} / H z\right)=2 e V R \operatorname{coth}\left(\frac{e V}{2 k_{B} T}\right)+\frac{\alpha V^{2}}{A f^{\gamma}},
$$

where $V$ is the bias voltage on the junction, $R$ is the junction resistance, and $A$ is the junction area. The first term is a
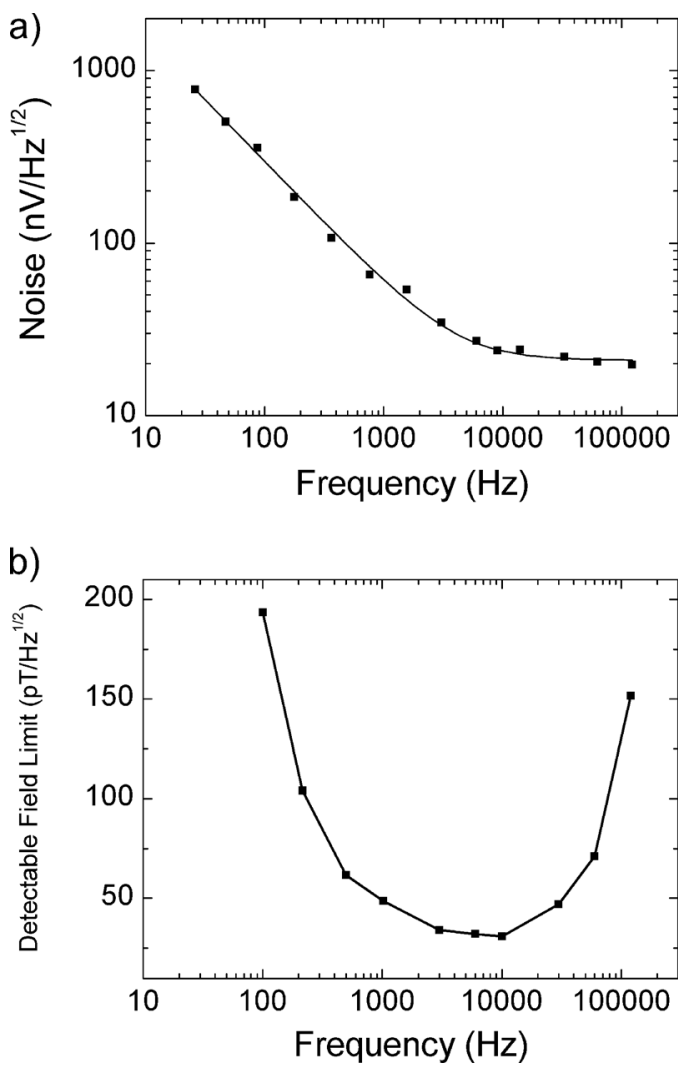

FIG. 4. (a) Noise spectrum for an MTJ sensor with double-staged MFCs, with the black solid line as theoretical noise fitting; and (b) detectable field limit calculated based on Fig. 3(c) and Fig. 4(a) as a function of frequency up to $100 \mathrm{kHz}$. combination of Johnson and shot noise and does not have any frequency dependence, while the second term is the electric $1 / f$ noise, characterized by the Hooge-like parameter $\alpha$ and exponent $\gamma$. Fitting of the sensor's noise power spectral density gives $\alpha=1.6 \times 10^{-7} \mu \mathrm{m}^{2}$ and $\gamma=1.43$. The relatively large value of $\alpha$ may be related to the high sensitivity of the MTJ sensor. The fitted white noise is $4.3 \times 10^{-16} \mathrm{~V}^{2} / \mathrm{Hz}$, slightly larger than the theoretical prediction of shot and Johnson noise $\left(3.0 \times 10^{-16} \mathrm{~V}^{2} / \mathrm{Hz}\right)$. Such enhancement of shot noise implies the presence of the spin-blockade effect, which has been observed in previous studies on MTJs. ${ }^{34-36}$

Combining the frequency-dependent response and noise spectrum of the MTJ sensor, we can calculate its detectable field limit as the ratio between noise and response. While the response decreases monotonically as the frequency increases, the noise decreases first and saturates at around $10 \mathrm{kHz}$. Figure 4(b) shows the detectable field from 0.1 to $120 \mathrm{kHz}$. At low frequency, the rise of $1 / f$ noise dominates over the increment of response, giving rise to a high detectable field limit of $195 \mathrm{pT} / \sqrt{\mathrm{Hz}}$ at $100 \mathrm{~Hz}$; at high frequency, the noise level becomes frequency-independent while response continues to drop. Therefore, the optimal performance of the MTJ sensor is achieved at intermediate frequencies $(3-30 \mathrm{kHz})$ with the detectable field limit going as low as $30 \mathrm{pT} / \sqrt{\mathrm{Hz}}$. This result is remarkable compared with recent developments of MTJbased sensors with MFCs which shows their best detectable field limits to be $50 \sim 100 \mathrm{pT} / \sqrt{\mathrm{Hz}}$ and coercive forces of $0.1 \sim 10 e^{8,23,27,37,38}$ However in these previous developments, the sensor's response has been assumed frequency-independent and determined from the transfer curve under the DC magnetic field. But the decrease in the sensor response at smaller fields and higher frequencies would lead to an increase in the detectable field limit. Therefore, our method of direct measurement of the high frequency sensor response is necessary in characterizing the full performance of the MTJ sensor.

In summary, we have developed double-staged magnetic flux concentrators which have enhanced the magnetic field sensitivity of the MTJ sensors to an unprecedented level. The first stage MFC is a shaped CoZrNb thin film, which we have optimized the fabrication process to acquire a field amplification factor of 18.8. This on-chip MFC is incorporated naturally on the MTJ sensor chip with a small footprint. The second stage MFC uses a "soft" alloy of $\mathrm{Ni}_{77} \mathrm{Fe}_{14} \mathrm{Cu}_{5} \mathrm{Mo}_{4}$ which is larger in size and is in contact with the on-chip MFC. This external MFC has an even larger amplification factor of 30.8. Together with a total amplification of 516.9, our MTJ sensor with dual MFCs sports a MR sensitivity of $775.4 \% / \mathrm{Oe}$ and an ultimate detectable field limit of $30 \mathrm{pT} / \sqrt{\mathrm{Hz}}$ at $10 \mathrm{kHz}$. We are already trying to apply this sensor to oil exploration, and working on shrinking the size of MFCs to make it more applicable to other fields. There is still much room for further improvement in our sensors. For example, the $1 / f$ noise of the MTJ sensors could be reduced further, and the dual MFCs can be made more magnetically permeable. It is likely that our technical approach could lead to femtoTesla level field sensitivity upon continuing improvement.

This work was supported by the National Science Foundation through Grant No. DMR-1307056 and by the 
King Abdullah University of Science and Technology (KAUST).

${ }^{1}$ G. Xiao, in Handbook of Spin Transport and Magnetism, edited by E. Tsymbal, I. Zutic (CRC Press, Taylor \& Francis, Boca Raton, 2012), pp 665-684.

${ }^{2}$ S.-H. Liou, X. Yin, S. E. Russek, R. Heindl, F. C. S. Da Silva, J. Moreland, D. P. Pappas, L. Yuan, and J. Shen, IEEE Trans. Magn. 47(10), 3740 (2011).

${ }^{3}$ P. P. Freitas, R. Ferreira, S. Cardoso, and F. Cardoso, J. Phys. Condens. Matter 19(16), 165221 (2007).

${ }^{4}$ D. Mazumdar, X. Liu, B. D. Schrag, W. Shen, M. Carter, and G. Xiao, J. Appl. Phys. 101(9), 09B502 (2007).

${ }^{5}$ J.-G. J. Zhu and C. Park, Mater. Today 9(11), 36 (2006).

${ }^{6}$ S. Ikeda, J. Hayakawa, Y. M. Lee, F. Matsukura, Y. Ohno, T. Hanyu, and H. Ohno, IEEE Trans. Electron Devices 54(5), 991 (2007).

${ }^{7}$ N. A. Stutzke, S. E. Russek, D. P. Pappas, and M. Tondra, J. Appl. Phys. 97(10), 10Q107 (2005).

${ }^{8}$ R. C. Chaves, P. P. Freitas, B. Ocker, and W. Maass, Appl. Phys. Lett. 91(10), 102504 (2007).

${ }^{9}$ W. F. Egelhoff, Jr., P. W. T. Pong, J. Unguris, R. D. McMichael, E. R. Nowak, A. S. Edelstein, J. E. Burnette, and G. A. Fischer, Sens. Actuators A: Phys. 155(2), 217 (2009).

${ }^{10}$ R. Ferreira, P. Wisniowski, P. P. Freitas, J. Langer, B. Ocker, and W. Maass, J. Appl. Phys. 99(8), 08 K706 (2006).

${ }^{11}$ W. Zhang, Q. Hao, and G. Xiao, Phys. Rev. B 84(9), 094446 (2011).

${ }^{12}$ D. Mazumdar, X. Liu, B. D. Schrag, M. Carter, W. Shen, and G. Xiao, Appl. Phys. Lett. 91(3), 033507 (2007).

${ }^{13}$ D. Mazumdar, W. Shen, X. Liu, B. D. Schrag, M. Carter, and G. Xiao, J. Appl. Phys. 103(11), 113911 (2008).

${ }^{14} \mathrm{P}$. Wisniowski, J. M. Almeida, and P. P. Freitas, IEEE Trans. Magn. 44(11), 2551 (2008).

${ }^{15}$ P. P. Freitas, S. Cardoso, R. Sousa, W. Ku, R. Ferreira, V. Chu, and J. P. Conde, IEEE Trans. Magn. 36(5), 2796 (2000).

${ }^{16}$ B. D. Schrag, M. J. Carter, X. Liu, J. S. Hoftun, and G. Xiao, paper presented at the International Symposium for Testing and Failure Analysis, 2006.

${ }^{17}$ E. A. Lima, A. C. Bruno, H. R. Carvalho, and B. P. Weiss, Meas. Sci. Technol. 25(10), 105401 (2014).
${ }^{18}$ J. W. Lau and J. M. Shaw, J. Phys. D: Appl. Phys. 44(30), 303001 (2011).

${ }^{19}$ M. N. Nabighian, V. J. S. Grauch, R. O. Hansen, T. R. LaFehr, Y. Li, J. W. Peirce, J. D. Phillips, and M. E. Ruder, Geophysics 70(6), 33ND (2005).

${ }^{20}$ X. Tao, L. Wusheng, L. Haibao, and L. Qin, Sens. Actuators A: Phys. 119(2), 427 (2005).

${ }^{21}$ W. Shen, X. Liu, D. Mazumdar, and G. Xiao, Appl. Phys. Lett. 86(25), 253901 (2005).

${ }^{22}$ W. Shen, B. D. Schrag, M. J. Carter, and G. Xiao, Appl. Phys. Lett. 93(3), 033903 (2008).

${ }^{23}$ X. Yin, Y.-F. Liu, D. Ewing, C. K. Ruder, P. J. De Rego, A. S. Edelstein, and S.-H. Liou, presented at the Spintronics VIII, 2015.

${ }^{24}$ J.-G. Choi, D.-G. Hwang, J.-R. Rhee, and S.-S. Lee, J. Magn. Magn. Mater. 322(15), 2191 (2010).

${ }^{25}$ T. Takahashi, M. Yoneda, and M. Naoe, Jpn. J. Appl. Phys. Part 1 28(3R), 379 (1989).

${ }^{26}$ T. Takahashi, N. Ikeda, and M. Naoe, J. Appl. Phys. 69(8), 5011 (1991).

${ }^{27}$ A. Guedes, J. M. Almeida, S. Cardoso, R. Ferreira, and P. P. Freitas, IEEE Trans. Magn. 43(6), 2376 (2007).

${ }^{28}$ M. Michiyori, K. Hisashi, M. Kiyoshi, and K. Morio, IEEE Trans. Magn. 24(5), 2215 (1988).

${ }^{29}$ S. Park, W. Park, Y. J. Kim, and T. Kim, Phys. Status Solidi A 201(8), 1640 (2004).

${ }^{30}$ Z. Marinho, S. Cardoso, R. Chaves, R. Ferreira, L. V. Melo, and P. P. Freitas, J. Appl. Phys. 109(7), 07E521 (2011).

${ }^{31}$ P. M. Drljača, F. Vincent, P.-A. Besse, and R. S. Popović, Sens. Actuators A: Phys. 97, 10 (2002).

${ }^{32}$ X. W. Li, C. Song, J. Yang, F. Zeng, K. W. Geng, and F. Pan, J. Magn. Magn. Mater. 315(2), 120 (2007).

${ }^{33}$ Y. Cao and C. Zhou, J. Magn. Magn. Mater. 324(10), 1832 (2012).

${ }^{34}$ B. R. Bułka, Phys. Rev. B 62(2), 1186 (2000).

${ }^{35}$ W. Belzig, Phys. Rev. B 71(16), 161301 (2005).

${ }^{36}$ A. Cottet, W. Belzig, and C. Bruder, Phys. Rev. Lett. 92(20), 206801 (2004).

${ }^{37}$ J. M. Almeida and P. P. Freitas, J. Appl. Phys. 105(7), 07E722 (2009).

${ }^{38}$ E. Paz, S. Serrano-Guisan, R. Ferreira, and P. P. Freitas, J. Appl. Phys. 115(17), 17E501 (2014). 